\title{
CONTRIBUIÇÃO AO ESTUDO DA SOLUBILIDADE DE ELETRÓLITOS EM SOLVENTES PUROS E MISTURAS DE SOLVENTES.
}

\author{
A. H. R. REZENDE ${ }^{1}$, L. C. P. BORGES ${ }^{1}$, M. R. FRANCO Jr² e R. F. PIRES ${ }^{1}$ \\ ${ }^{1}$ Universidade Federal do Triângulo Mineiro, Departamento de Engenharia Química \\ ${ }^{2}$ Universidade Federal de Uberlândia, Faculdade de Engenharia Química \\ E-mail para contato: fpires.ricardo@gmail.com
}

\begin{abstract}
RESUMO - O equilíbrio solido-liquido está presente em várias aplicações industriais. Neste preâmbulo, o conhecimento de dados de solubilidade de um eletrólito num solvente ou mistura de solventes é muito importante. Neste trabalho, foram medidas solubilidades do ácido succínico e da tiamina em solventes puros e misturas binárias de solventes. Os experimentos foram conduzidos à pressão atmosférica numa faixa de temperatura de $298,15 \mathrm{~K}$ a $323,15 \mathrm{~K}$. O procedimento experimental consistiu, basicamente, em alimentar o eletrólito e os solventes de forma a preparar a mistura sólido-líquido dentro da célula. Depois, a mistura era agitada e em seguida decantada. Finalmente, apenas a fase líquida era amostrada utilizando seringa de vidro. As amostras foram analisadas por gravimetria. Foram feitos ajustes dos dados obtidos, nas condições supracitadas, a modelos empíricos disponíveis na literatura. Resultados de solubilidade eletrólito-solvente mostraram boa concordância com valores fornecidos pela literatura.
\end{abstract}

\section{INTRODUÇÃO}

O desenvolvimento e aprimoramento de processos industriais envolvem necessariamente um pleno conhecimento da matéria-prima alvo do beneficiamento ou transformação a ser empreendida. Para tanto, a informação acerca dos dados de solubilidade é extremamente valiosa, pois permite, nas condições de operação desejadas, garantir a concentração almejada de um eletrólito num dado solvente; ou ainda, mediante o uso de cossolventes, garantir a proporção desejada do soluto de interesse. Desta forma, pode-se controlar uma maior ou menor quantidade de eletrólito variando condições como pressão, temperatura e percentagem de solventes na mistura.

Solutos como ácidos orgânicos e proteínas são, nesse contexto, um bom material de estudo para a determinação de solubilidade utilizando misturas de solventes orgânicos e água, pois são altamente empregados numa grande variedade de processos industriais além de fazerem parte do cotidiano da sociedade. Como representantes dessas duas classes de substâncias têm-se o ácido succínico e a vitamina B1, conhecida como tiamina.

A vitamina B1, ou tiamina, é uma das vitaminas pertencentes ao complexo B, e pode ser 
encontrada na cutícula do arroz, no levedo de cerveja, nos grãos, na gema de ovo, no fígado, no rim, na carne de porco, no peixe, no amendoim, na noz, nos legumes, e em vegetais verdes e folhosos. Atua em diversas partes do organismo exercendo um importante papel no sistema nervoso, muscular e cardíaco, além de auxiliar no metabolismo da glicose.

A carência da tiamina provoca lesão cerebral, que em alguns casos pode ser irreversível. Entre as doenças provocadas pela deficiência de vitamina B1 temos: Beribéri, Encefalopatia de Wernicke e Síndrome de Korsakoff (HARPER, 1983).

A tiamina não é armazenada em grande quantidade no organismo, sendo necessário seu fornecimento diário pela dieta. Trata-se de um composto instável ao calor em álcali e estável ao calor em pH ácido.

O ácido succínico também chamado de ácido butanodióico é um ácido dicarboxílico de grande importância na indústria química em geral. Este composto participa do metabolismo energético em todas as células animais e vegetais como um intermediário do ciclo de Krebs, ou ciclo do ácido cítrico.

O ácido succínico é um precursor versátil na produção de outros diferentes ácidos carboxílicos e ésteres, bem como de inúmeros outros produtos. Em se tratando de alimentos, é utilizado na produção de bebidas como refrigerantes e cervejas, serve como aromatizante e neutralizante, e é produzido pela fermentação de carboidratos. Já na indústria de medicamentos, é empregado na preparação de agentes que combatem a úlcera, sendo também utilizado como um suplemento nutricional. (ZEIKUS, 1999)

Dados de solubilidade do ácido succínico e da tiamina em água são facilmente disponíveis. Por outro lado, tais dados de solubilidade em outros solventes (DMF e DMSO) e em misturas aquosas (DMF + água; DMSO + água) nem sempre estão ao alcance. Temos por exemplo os estudos de NETO et al., (2010) e PIRES et al.,(2006) .Tem-se que lembrar a importância destes dados em estudos de processos de separação e purificação destes insumos. Infelizmente, existem poucos dados de solubilidade destes importantes insumos que mostrem a relação de dependência desta solubilidade com a temperatura juntamente com a concentração da mistura inicial de solventes. Sendo assim, a obtenção de dados de solubilidade do ácido succínico e da tiamina em condições conhecidas de temperatura, pressão e concentração da mistura inicial de solventes é importante na melhora dos processos de fabricação e purificação (cristalização) destes produtos assim como na melhoria dos processos existentes.

\section{MATERIAIS E MÉTODOS}

\subsection{Compostos Químicos e Sistemas Estudados}

Os solutos usados no presente trabalho foram utilizados em 2 sistemas, que estão descritos na Tabela 1, enquanto que a relação dos reagentes utilizados no trabalho encontra-se disposta na Tabela 2, juntamente com o fabricante e a pureza referente a cada um deles. 
19 a 22 de outubro de 2014
Florianópolis/SC

Tabela 2- Especificações dos Reagentes.

\begin{tabular}{|c|c|c|}
\hline Compostos & Fabricante & Pureza (\%) \\
\hline Ac. succínico & ISOFAR & $99 \%$ \\
\hline Tiamina & Synth & $99 \%$ \\
\hline DMF & Vetec & $99,8 \%$ \\
\hline DMS & Vetec & $99,9 \%$ \\
\hline & & \\
\hline
\end{tabular}

O sistema 1 foi analisado variando as concentrações de DMF em água (0,5,10,25 e 100\%), enquanto que o sistema 2 foi estudado com as concentrações de água em DMSO (0,2,5 10 e 20\%). Esses valores foram escolhidos vislumbrando a obtenção de dados ainda não disponíveis na literatura. As temperaturas utilizadas nos experimentos foram $298,15 \mathrm{~K}$ a $323,15 \mathrm{~K}$ com variação de $5 \mathrm{~K}$.

\subsection{Aparato Experimental}

Com a finalidade de determinar experimentalmente dados de solubilidade de um eletrólito para sistemas isotérmicos variando as frações de mistura de solventes, uma unidade experimental foi desenvolvida e montada conforme a Figura 1.

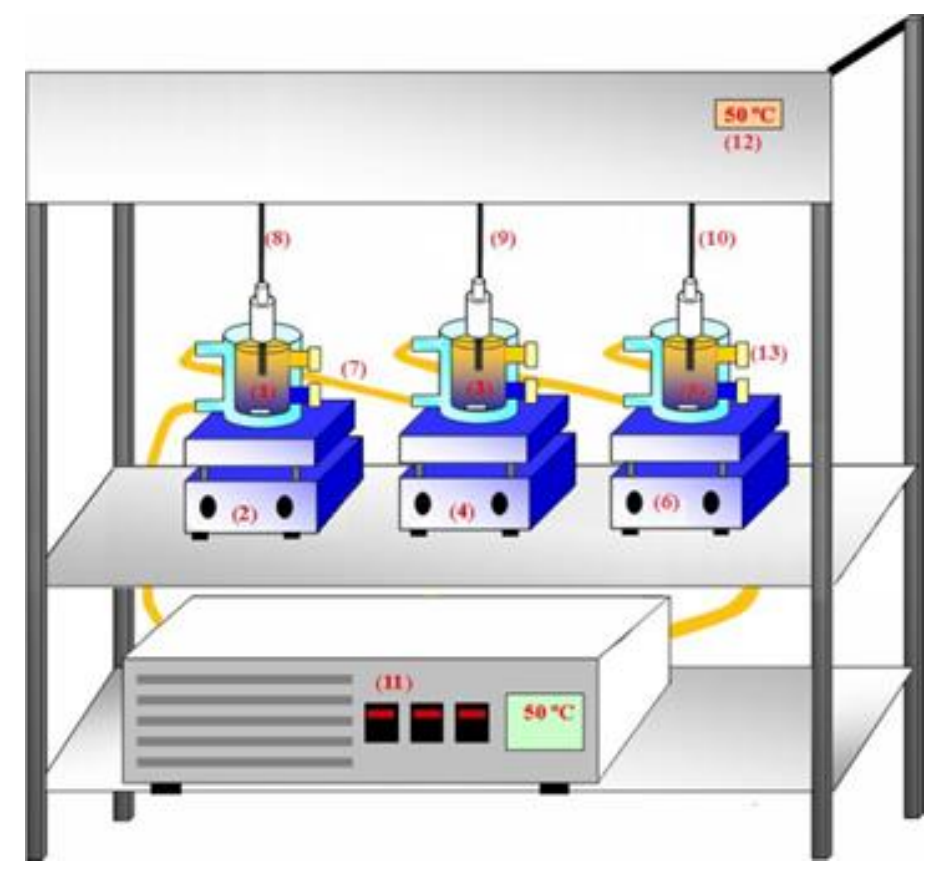

Figura 1. Aparato Experimental. 1, 3 e 5. Células de Equilíbrio; 2, 4 e 6. Agitadores Magnéticos; 8, 9 e 10. Termômetros; 7. Mangueiras de látex; 13. Septos; 11. Banho Termostático; 12. Indicadores de temperatura.

O equipamento experimental empregado nesse trabalho constituiu de células encamisadas de 
vidro pirex, em que na maioria dos experimentos foram conectadas em série, interligadas por mangueiras de látex e conectados a um banho termostático da marca CIENLAB, o qual mantinha a temperatura do sistema constante (precisão $\pm 0,1^{\circ} \mathrm{C}$ ). As células foram apoiadas sobre agitadores magnéticos da marca edutec modelo EEQ-9010. As células de equilíbrio eram constituídas de uma camisa externa na qual circulava uma massa água provida do banho termostático previamente conectado às células. $\mathrm{Na}$ parte superior das células foram fixadas rolhas que tinham por finalidade vedar o sistema e apoiar o termômetro utilizado para controlar a temperatura da solução.

\subsection{Procedimento Experimental}

Primeiramente as células foram devidamente higienizadas e, posteriormente dispostas na configuração já descrita no aparato experimental. As misturas de solventes foram inseridas nas células de acordo com as concentrações adotadas e, após a estabilização da temperatura oferecida pelo banho termostático os solutos foram então adicionados em excesso para garantir que a entropia máxima necessária para o estado de equilíbrio fosse alcançada.

O procedimento experimental consistiu em uma agitação com o período de 162 minutos, seguidos por uma decantação de 180 minutos. Ao final do processo de agitação e repouso da solução, as amostras eram coletadas por meio de uma seringa termostatizada de $5 \mathrm{~mL}$. Foi tomado o devido cuidado para que apenas o seio do fluido fosse amostrado, não retirando sólidos precipitados no fundo da célula em questão. Para cada solução foram retiradas três amostras, que posteriormente foram analisadas gravimetricamente.

As análises gravimétricas foram feitas utilizando uma estufa fabricada por Biodont, uma balança analítica (precisão 0,0001) da marca Edutec, modelo FA2204C e um dessecador de vidro.

\subsection{Ajustes computacionais}

Os trabalhos de Pires (2007), (Pires et al. 2008) e Pires (2011) mostram que o uso de equações empíricas para correlação de dados de equilíbrio sólido-líquido é de grande utilidade. A fim de verificar a possibilidade da correlação dos dados de solubilidade, a partir de equações empíricas publicadas na literatura que correlacionam dados de solubilidade em função da temperatura, foram utilizadas as equações desenvolvidas por HEIDMAN et al.(1985) e YAWS et al.(1993). Estas foram modificadas e adequadas para o ajuste aos dados deste trabalho. As equações originais desenvolvidas por HEIDMAN et al.(1985) (1) e YAWS et al.(1993) (2) são apresentadas:

$$
\begin{aligned}
& \ln m=A+\frac{B}{T}+C \ln T \\
& \log m=A^{\prime}+\frac{B^{\prime}}{T}+\frac{C^{\prime}}{T^{2}}
\end{aligned}
$$


Sendo m, a molalidade do eletrólito na solução e T, a temperatura, em Kelvin, da mistura sólidolíquido dentro da célula de equilíbrio. Observando as Equações 1 e 2 propostas pelos autores, nota-se que são ajustes que levam em conta apenas a variável temperatura como fator determinante na solubilidade do soluto em questão. Com o intuito de introduzir nas equações, os efeitos da concentração da mistura inicial de solventes, livre de soluto, juntamente com o efeito da temperatura, foram feitas modificações.

As constantes $A$ e $A^{\prime}$ das Equações 1 e 2, foram substituídas por $A^{\mathrm{h}}$ e $A^{\mathrm{y}}$ respectivamente. As constantes B e B' passaram a ser $B^{h}$ e $B^{y}$, respectivamente. E por fim, as constantes $C$ e $C^{\prime}$ foram substituídas por $C^{h}$. $C_{S O L V}$ e $C^{y} . C_{S O L V}$, (em que $C_{S O L V}$ refere-se à concentração do solvente 1 ou 2 na mistura de solventes inicial livre de eletrólito) respectivamente. Segundo as modificações feitas obtém-se as Equações 3 e 4, que ficaram da seguinte forma:

$\ln m=A^{h}+\frac{B^{h}}{T}+C^{h} \cdot C_{S O L V} \cdot \ln T$

$\ln m=A^{y}+\frac{B^{y}}{T}+\frac{{ }^{y} \cdot C_{S O L V}}{T^{2}}$

Na obtenção destes parâmetros foi feita uma otimização através do uso do programa STATISTICA 7.0. A função objetivo empregada foi a minimização dos quadrados dos resíduos e o método utilizado na estimação dos parâmetros foi Levenberg Marquardt, com 500 iterações e precisão da ordem de $10^{-6}$.

\section{RESULTADOS E DISCUSSÃO}

\subsection{Resultados Experimentais}

As Tabelas 3 e 4 juntamente com as Figuras 1 e 2 demonstram os resultados obtidos experimentalmente para a solubilidade do ácido succínico em misturas de água e DMF e da Tiamina em misturas de água e DMSO, respectivamente. 
Tabela 3: Dados da molalidade do ácido succínico em misturas de água e DMF.

\begin{tabular}{|l|l|l|l|l|l|}
\hline \multirow{3}{*}{$\mathrm{T}(\mathrm{K})$} & \multicolumn{5}{|c|}{$C_{2}$} \\
\cline { 2 - 6 } & 0,00 & 5,0534 & 10,0416 & 25,0382 & 100,00 \\
\cline { 2 - 6 } & \multicolumn{5}{|c|}{$\mathrm{m}_{1}\left(\mathrm{~mol}_{\mathrm{kg}}{ }^{-1}\right)$} \\
\hline 298,15 & 0,7120 & 0,8213 & 0,9392 & 1,5592 & 4,9913 \\
\hline 303,15 & 0,8767 & 1,0199 & 1,1407 & 1,6602 & 5,7210 \\
\hline 308,15 & 1,0655 & 1,2330 & 1,4317 & 2,0469 & 6,1653 \\
\hline 313,15 & 1,2992 & 1,4923 & 1,6938 & 2,4460 & 6,1046 \\
\hline 318,15 & 1,5558 & 1,7935 & 2,0262 & 2,7872 & 6,3726 \\
\hline 323,15 & 1,8520 & 2,1728 & 2,5620 & 3,1396 & 7,2018 \\
\hline
\end{tabular}

Tabela 4. Dados de molalidade da tiamina em misturas DMSO e água.

\begin{tabular}{|c|c|c|c|c|c|}
\hline \multirow{2}{*}{$\mathrm{T}(\mathrm{K})$} & \multicolumn{5}{|c|}{$\mathrm{C}_{1}$} \\
\cline { 2 - 6 } & 0,00 & 2,00 & 5,00 & 10,00 & 20,00 \\
\cline { 2 - 6 } & \multicolumn{5}{|c|}{$\mathrm{m}_{2}\left(\mathrm{~mol}_{\mathrm{kg}} \mathrm{-}^{-1}\right)$} \\
\hline 298,15 & 0,0574 & 0,0655 & 0,0815 & 0,1007 & 0,1892 \\
\hline 303,15 & 0,0585 & 0,0659 & 0,0729 & 0,1037 & 0,1875 \\
\hline 308,15 & 0,0691 & 0,0696 & 0,0824 & 0,1175 & 0,2028 \\
\hline 313,15 & 0,0762 & 0,0748 & 0,0871 & 0,1285 & 0,2375 \\
\hline 318,15 & 0,0812 & 0,0715 & 0,1131 & 0,1532 & 0,2445 \\
\hline 323,15 & 0,0833 & 0,0941 & 0,1112 & 0,1671 & 0,2924 \\
\hline
\end{tabular}

Através da Figura 3 pode-se perceber que, em todas as concentrações estudadas, a solubilidade $\left(\mathrm{m}_{2}\right)$ da tiamina tende a aumentar com o incremento da temperatura. Tal comportamento fora observado por outros pesquisadores (Oliveira et al., 2007; Jiang et al., 2013). Fazendo um adendo, observa-se pela Tabela 4 que numa mesma temperatura o aumento do solvente 1 (água) na mistura inicial de solventes promove o incremento da solubilidade da tiamina.

Em relação à solubilidade $\left(\mathrm{m}_{1}\right)$ do ácido succínico, observa-se por meio da Tabela 3 que a solubilidade do eletrólito analisado aumenta com o incremento da concentração $\left(\mathrm{C}_{2}\right)$ do DMF em um dado sistema isotérmico. Somando a isso, é possível analisar pela Figura 2 que ao fornecer temperatura para o sistema, e manter fixa a concentração $C_{2}$, a solubilidade $\left(\mathrm{m}_{1}\right)$ do ácido succínico se 


\section{9 a 22 de outubro de 2014}

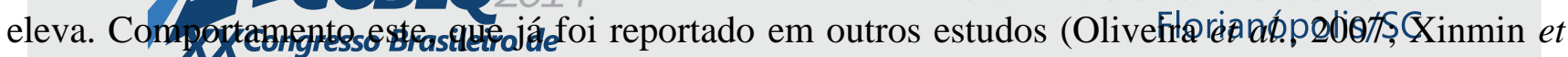
al., 2012). Engenharia Química

Os resultados dos parâmetros, dos desvios padrões dos mesmos e dos coeficientes de correlação para os ajustes referentes às equações 3 e 4 aplicados aos sistemas 1 e 2 estão reportados na Tabela 5.

Tabela 5. Resultados dos parâmetros dos ajustes 3 e 4 para os sistemas ácido succínico+água(1)+DMF(2) e tiamina+água(1)+DMSO(3).

\begin{tabular}{|c|c|c|c|c|c|c|c|}
\hline & Sistema & \multicolumn{3}{|c|}{ Ajuste 3} & \multicolumn{3}{|c|}{ Ajuste 4} \\
\hline \multirow[t]{5}{*}{1} & \multirow{5}{*}{$\begin{array}{c}\text { Ácido succínico + } \\
\text { Água (1) + } \\
\text { DMF (2) }\end{array}$} & \multicolumn{2}{|c|}{ Parâmetro } & Desvio & \multicolumn{2}{|c|}{ Parâmetro } & \multirow{2}{*}{$\begin{array}{c}\text { Desvio } \\
0,751\end{array}$} \\
\hline & & $A^{h}$ & 10,2 & 0,917 & $A^{y}$ & 11,2 & \\
\hline & & $B^{h}$ & $-3,09.10^{3}$ & $2,83 \cdot 10^{2}$ & $B^{y}$ & $-3,40.10^{3}$ & $2,33 \cdot 10^{2}$ \\
\hline & & $C^{h}$ & $3.10^{-3}$ & $1.10^{-4}$ & $C^{y}$ & $1,51 \cdot 10^{3}$ & $5,22.10^{1}$ \\
\hline & & \multicolumn{3}{|c|}{$\mathrm{R}^{2}=0,962$} & \multicolumn{3}{|c|}{$R^{2}=0,975$} \\
\hline \multirow[t]{4}{*}{2} & \multirow{4}{*}{$\begin{array}{l}\text { Tiamina + } \\
\text { Água(1) + } \\
\operatorname{DMSO}(3)\end{array}$} & $A^{h}$ & 2,50 & 0,470 & $A^{y}$ & 3,500 & 0,530 \\
\hline & & $B^{h}$ & $-1,61.10^{3}$ & $1,47 \cdot 10^{2}$ & $B^{y}$ & $-1,91 \cdot 10^{3}$ & $1,64 \cdot 10^{2}$ \\
\hline & & $C^{h}$ & 1,0 & $3,2.10^{-2}$ & $C^{y}$ & $5,73.10^{5}$ & $1,96.10^{4}$ \\
\hline & & \multicolumn{3}{|c|}{$\mathrm{R}^{2}=0,978$} & \multicolumn{3}{|c|}{$\mathrm{R}^{2}=0,978$} \\
\hline
\end{tabular}

Os desvios relativos médios, para o sistema 1 foram $1,067 \%$ e $0,858 \%$ para os ajustes 3 e 4 , respectivamente; enquanto que para o sistema 2 os valores obtidos foram $0,054 \%$ e 4,261\%. Verificase neste caso uma média dos desvios relativos menores que 5\%, o que denota pequenas variações entre os resultados correlacionados pelo modelo e os obtidos experimentalmente. Por sua vez, os coeficientes de correlação para os ajustes 3 e 4 mostraram ajustes maiores que 96,0\%. Diante do exposto, percebe-se que ambos os ajustes prestam-se igualmente para a correlação/predição dos dados experimentais obtidos em laboratório nas condições especificadas.

\section{CONCLUSÕES}

Os resultados obtidos experimentalmente para a solubilidade do ácido succínico em misturas de solventes (água+DMF) utilizando gravimetria relatam que em um sistema isotérmico, a solubilidade do eletrólito em questão aumenta com o incremento da concentração $\left(\mathrm{C}_{2}\right)$ na mistura de solução. Além do mais, os dados obtidos indicam um aumento da solubilidade do ácido à medida que a temperatura é elevada, entretanto a proporcionalidade dos incrementos variou para diferentes soluções. Por fim, conclui-se que a solubilidade experimental e os parâmetros calculados pelo modelo HEIDMAN et al.(1985) e YAWS et al.(1993) neste estudo podem ser utilizados na indústria para a purificação do ácido succínico em diferentes misturas dos solventes (1) e (2). Os experimentos realizados obtiveram resultados satisfatórios se comparados aos resultados obtidos em (Xinmin et al., 2012).

O caso da solubilidade da tiamina em mistura de solventes (água+DMSO) retrata, para um sistema isotérmico, que a solubilidade da vitamina é muito alta em água (soluto altamente higroscópico); ou seja, apresenta um aumento diante do incremento da concentração de água como co-solvente em mistura com DMSO. Comportamento comum entre vitaminas como o observado em FONSECA et.al.(2013). Somado a isso, observa-se que assim como o ácido succínico, a tiamina satisfaz a regra geral de solubilidade que implica no aumento da solubilidade diante do acréscimo de 


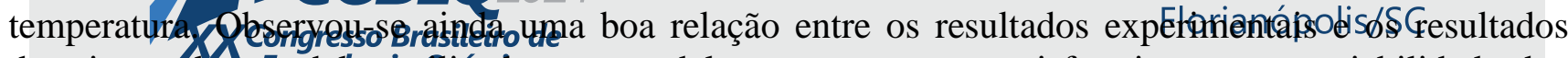
descritos pelos ifresellogriatQúźados; os modelos representaram satisfatoriamente a variabilidade dos dados obtidos de forma experimental.

\section{NOMENCLATURA}

$\mathrm{C}_{1}$ :Concentração de água;

$\mathrm{C}_{2}$ :Concentração de DMF;

$\mathrm{C}_{3}$ :Concentração de DMSO;

$\mathrm{m}_{1}$ :Molalidade do ácido succínico.

$\mathrm{m}_{2}:$ Molalidade da tiamina.

\section{REFERÊNCIAS}

FONSECA, L. G.; OLIVEIRA, F.; SILVA, C. V.; PIRES, R. F.; MALAGONI, R. A. Influência da temperatura na solubilidade de alfa-tecoferol em etanol+água. In: XVIII JORNEQ - Jornada em Engenharia Química, 2013, Uberlândia - MG. . Anais da XVIII JORNEQ, 2013. Uberlândia - MG, 2013. p. 1-4.

HARPER, C. G. The incidence of Wernicke's encephalopathy in Australia: a neuropathological study of 131 cases. J. Neurol. Neurosurg. Psychiatr. v. 46, p. 593-598, 1983.

JIANG, X.; HU, Y.; MENG, Z.; YANG, W.; SHEN, F. Solubility of succinic acid in different aqueous solvent mixtures: Experimental measurement and thermodynamic modeling. Fluid Phase Equilibria, v. 341, p. 7-11, 2013.

NETO, A. C. R.; FRANCO JR, M. R.; PIRES, R. F.; MALAGONI, R. A. Solubility of Vitamin C in Water, Ethanol, Propan-1-ol, Water + Ethanol, and Water + Propan-1-ol at (298.15 and 308.15) K. J. of Chem. and Eng. Data, v. 55, p. 1718-1721, 2010.

OLIVEIRA, A. C.; PIRES, R. F.; COELHO, M. G.; FRANCO JR, M. R. Solubility of Benzoic Acid in Mixed Solvents. J. of Chem. and Eng. Data, v. 52, p. 298-300, 2006.

PIRES, R. F.; FRANCO JÚNIOR, M. R. solubility of salicylic acid in water +salt ( $\mathrm{NaCl}, \mathrm{KCl}, \mathrm{NaBr}$, $\mathrm{Na}_{2} \mathrm{SO}_{4}$ and $\mathrm{K}_{2} \mathrm{SO}_{4}$ ) at 293.5-313.15K. Fluid Phase Equilibria, 2012.

ZEIKUS, J. G.; JAIN, M. K.; ELANKOVAN, P. "Biotechnology of succinic acid production and markets for derived industrial products". Appl. Microbiol. and Biotech. v. 51, p.541-545, 1999.

\section{AGRADECIMENTOS}

Os autores agradecem o apoio financeiro provido pela FAPEMIG (PCE-00201-14: Participação Coletiva em Eventos Técnico-Científicos). 